\title{
Reflections from EASD 2015
}

\section{Dr Caroline Day reports from the European Association for the Study of Diabetes (EASD) conference in Stockholm, Sweden, 14-18th September, 2015}

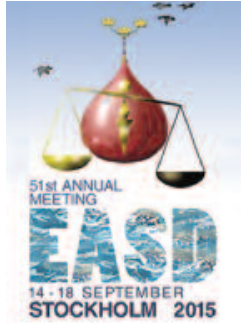

worsening heart failure or causing pancreatic cancer (/2045). The Evaluation of LIXisenatide in Acute coronary syndrome (ELIXA) session affirmed the safety of lixisenatide in type 2 diabetes as reported at ADA 2015 (/2046).

Post-hoc analysis of data from the Examination of cardiovascular outcomes: Alogliptin versus standard of care (EXAMINE) trial revealed no influence of $A C E$ inhibition on the CV safety of alogliptin relative to placebo (\#822). In Saxagliptin Assessment of Vascular Outcomes Recorded in patients with diabetes mellitus (SAVOR)TIMI, health related quality of life (HRQoL) was decreased by CV events but saxagliptin (which increased hospitalisation for heart failure) did not alter HRQoL compared with other treatments (\#1188).

\section{Safety}

A Bayesian meta-analysis of survival data suggested a higher risk of CV events with sulphonylureas than with other glucoselowering agents (\#128) and a Swedish database study has shown that second line treatment with a sulphonylurea compared to a DPP4 inhibitor increased CV risk (\#129). A database study from the USA in type 2 diabetes patients with CV disease starting a DPP4 inhibitor or a sulphonylurea $(27,259$ in each cohort) showed no increase in hospitalisation for heart failure for either class of agent and specifically between saxagliptin and sitagliptin (\#370).

According to meta-analyses, dulaglutide (\#77), liraglutide (\#1193) or the novel basal insulin peglispro (\#132) did not increase MACE in type 2 diabetes. Analyses of pooled data showed that treatment with dapagliflozin did not increase CV events in elderly, hypertensive type 2 diabetes patients with coronary heart disease (\#754) or heart failure (\#765).

An overview of the DUAL I-V trials (DUAL $\mathrm{VI}$ and VII are ongoing) with a fixed-ratio combination injection (IDegLira; insulin degludec and liraglutide) showed no increase in pancreatitis (/2042). A nationwide database study in Denmark found no increased risk of pancreatic cancer with GLP-1RA and DPP4 inhibitors; however type 2 diabetes itself is a risk factor (\#17). Special sessions considered the conse- quences of hypoglycaemia (/2019) and trending topics in SGLT2 inhibition (/2170), particularly with regard to diabetic ketoacidosis in type 1 diabetes.

\section{Glucocentric}

The Swedish National Diabetes Registry has shown that type 2 diabetes still carries excess risk of all-cause and CV mortality, particularly in younger patients and those with poorer glycaemic control (\#271). Approaches to glucose lowering were discussed in the EASD/European Society of Cardiology Symposium (/2010) and the case made for early pathologically-guided intensive glycaemic control (/2025).

\section{Diary date}

EASD 2016 will be held at the ICM Messe in Munich, 12-16th September. Early booking is recommended, or reserve your seat on the sofa for the virtual meeting.

\section{Acknowledgement}

Nobody can be in two places at once, so thanks go to Dr Mike Gwilt for pointing me towards some of the content described above.

\section{References}

1. Abstract book, EASD 2015. Diabetologia 2015; 58:S1-S607.

2. EASD virtual meeting website. www.easdvirtualmeeting.org

3. Whole sessions for online viewing can be accessed as follows:

http://www.easdvirtualmeeting.org/contentsessions/ followed by the reference given above, e.g.

http://www.easdvirtualmeeting.org/contentsessions/2030 takes you to the EMPAREG OUTCOMETM session.

4. The EASD programme flipbook is available at http://www.easd.org/images/easdwebfiles/an nualmeeting/51stmeeting/Flipbook/index. html

Dr Caroline Day

Visiting Fellow, Diabetes Group, Aston University, Birmingham, B4 7ET, UK. E-mail: cday@mededuk.com

http://dx.doi.org/10.15277/bjdvd.2015.051 Br J Diabetes Vasc Dis 2015;15:196 which confirm that sitagliptin can be safely used in type 2 diabetes without concern for 